\title{
Biomedical Informatics for Sustainable Health Systems
}

\author{
J.-C. Healy \\ UN Global Alliance for Information and Communication Technologies and Development (GAID), \\ Senior Advisor (New York)
}

All the present traditional healthcare systems are, or will shortly be, in crisis: We'll have an economical crisis, accelerated by the ageing of the populations, a medical crisis, due to the ethical issues and the "total quality" approach requested by the patients, an organisational crisis due to the lack of professionals for responding to the demands and finally a social crisis due to the gradual decrease of confidence of the patients in the efficiency of scientifically based medical approaches.

It is the responsibility of all the participants in the value chain, starting from the academic community up to politicians and to healthcare professionals, to anticipate this crisis by an indepth analysis of the future of the demands in health domains and the future of the individual and collective potential responses.

Based on history, we can easily anticipate the following demands:

Increasingly more demands will move towards a "citizen" demand, requesting to stay healthy at home, in the working place, in school etc. and no longer to be seen just as a "patient" demanding a cure for his or her diseases. Accordingly, education, prevention, assistance, early detection and an altogether global and personalised approach will be requested and be crucial.

More and more the present infrastructure and the way of working of the $\mathrm{HC}$ professionals will move from hospital disease-centred approaches towards a regional/national/international network of competences in the reflecting the diversity of languages, habits and personal requirements, and with optimization in the use of advanced resources. In brief, we can plan to move from the present "patient-centred, hospital-based healthcare" delivery system towards a "citizen-centred, health" delivery systems;

By chance we have now the conceptual and technical instruments needed for the implementation of this strategy; they are the Information and Communication technologies (ICT) for health, in short "eHealth". Of course, the use of ICT for productivity gain or saving money or time in the present $\mathrm{HC}$ delivery system is welcome, but more importantly, eHealth must be perceived as the backbone of the upcoming "citizen centred health systems". This vision changes radically the approach for researchers, politicians, health professionals and the ultimate users - the citizens, patients and their families.

This view is gradually more and more understood and accepted by both the rich and the not so rich countries. The role of the various traditional healthcare participants will be redesigned, new types of ICT based health jobs will be offered, new infrastructures will be needed specially for security purposes, but at the end the health sector will be perceived as one of the major beneficiaries of the globalisation of the knowledge economy.

This vision is not just a futuristic dream but already many new dynamic coun- 
tries offer to respond to the huge health demand of their new middle class by offering eHealth services (telemedicine, internet services, telemonitoring services etc.) for helping professionals, patients, and citizens.

Let the Biomedical Informatics Medical Community be a driving force for this substantial crucial global change, by a scientifically systematic approach to the new health problems. Some key new research topics can be mentioned:

- The personalized health approach is crucial: it is already implemented for treatment and drug delivery but the scope is larger, from genetic background towards daily personalized guidelines and codes of conduct. Of course the role of ICT will be crucial for action in the domain of predictive medicine, early detection, healthy habits guidelines, plans for supporting populations at risk, etc. Numerous versions or clones of products and services have to be developed. A specific clone of products will design the personalized way of learning and teaching based on the intellectual skills, the memory capacity and the personality of an individual.

- The digital modelling of the kinetics of biomolecules, of cells and cell interactions, of organs, of organisms and ecosystems, require huge collaborative efforts and substantial computer resources, now becoming possible by grid technologies. Why shouldn't the biologist duplicate the efficient collaborative work of nuclear scientists and astrophysics researchers using large expensive technical platforms together, coauthoring the published papers? The lab of biologists is all of humanity, and the target is not just knowledge, but action also.

- The systemic and dynamic approach, in quantitative terms, of the citizen centred health systems including psychological, social and economical components, will become a must. Of course the neurosciences will provide substantial inputs but a global approach for the network is needed.

The Healthcare delivery was the driving force of the last two (XIXth and XXth) centuries.

Public health will be a major issue of the XXIst century, not just for communicable diseases or epidemics, but for the day to day processes of health delivery. All states, both rich and not so rich, are equally affected and involved.

This challenge is global and applies to all the Nations of the World. No robust alternative exists and the worldwide health demand is growing faster than the present traditional responses. The solution of the problem is not just money-dependent but more urgently dependent of a new vision and implementation guidelines. It is in line with the outputs of the previous World Summit on an Information Society proposing to offer ICT facilities to all the citizens of the planet in the next ten years. Finally, by duplicating the famous outdated WHO Alma Ata declaration "Health for all by 2000", we can support the Millennium Development Goals (MDG) and dream of "eHealth for all by 2015" [1, 2].

This goal is achievable and it is our joint responsibility.

\section{References}

1. Healy JC. The WHO eHealth Resolution - eHealth for all by 2015? Methods Inf Med 2007;46:2-4.

2. World Health Organization . eHealth Resolution. 58th World Health Assembly, Resolution 28. May 25th, 2005. Geneva: WHO; 2005. 58th World Health Assembly's Homepage: htto:77www.who.int/gb/e/ e wha58.html.

English version: http://www.who.int/gb/ebwha/ pdf_files/WHA58/WHA58_28-en.pdf. Last accessed June 23, 2007

\section{Correspondence to:}

Prof. J.-C. Healy

UN Global Alliance for Information

and Communication Technologies and Development (GAID),

Senior Advisor (New York)

E-mail: jean-claude.healy@ec.europa.eu 